\title{
SOLUÇÕES ESTÉTICAS PARA PRÓTESES PARCIAIS REMOVÍVEIS RETIDAS POR GRAMPOS METÁLICOS
}

\author{
Guilherme Andrade Meyer Professor Assistente da \\ Universidade Federal da Bahia. \\ Francisco Barbosa de Oliveira Júnior \\ Cirurgião-Dentista - Universidade \\ Estadual do Sudoeste da Bahia. \\ Luciana Valadares Oliveira \\ Professora Adjunto da \\ Universidade Federal da Bahia. \\ Bruno Peixoto de Souza \\ Cirurgião-Dentista - Escola \\ Bahiana de Medicina e Saúde \\ Pública. \\ Felipe Vilasboas Rodrigues \\ Acadêmico do Curso de \\ Odontologia da Escola Bahiana de \\ Medicina e Saúde Pública. \\ Emilena Maria Castor Xisto Lima \\ Professora Adjunto da \\ Universidade Federal da Bahia.
}

Endereço para correspondência: gui.meyer@uol.com.br

\begin{abstract}
Resumo
A construção de uma Prótese Parcial Removível (PPR) tem por finalidade a reposição das unidades dentárias perdidas, incorporando-as ao sistema estomatognático, sem ferir o equilíbrio biomecânico, além do grande desafio, na atualidade, de aliar a função à estética. A dificuldade em se conseguir harmonia estética pela presença dos grampos em metal nas superfícies vestibulares de dentes anteriores justifica estudos que visam solucionar esse problema das PPRs, e ainda, o fato de se tratar de procedimento mais simples e menos oneroso para o paciente frente a outras opções de tratamento. Logo, o presente estudo apresentou soluções estéticas para PPR, propondo a comparação de grampos metálicos convencionais na face vestibular com grampos retentores na palatina, através de três casos clínicos de pacientes que apresentavam ausência parcial de unidades dentárias anteriores. Foram confeccionadas duas peças protéticas para cada paciente: 1) enfatizando apenas os princípios biomecânicos e 2) considerando princípios biomecânicos e estéticos. Observou-se que os grampos MDL modificado e Quereilhac são alternativas viáveis para atender às condições em PPRs de indivíduos desdentados anteriores, cujos arcos são Classe III e IV de Kennedy. Desta forma, foi possível constatar que houve melhora estética do sorriso em indivíduos parcialmente desdentados na região anterior, por meio da não visualização de estrutura metálica na face vestibular dos dentes pilares.
\end{abstract}

Palavras-chaves: Prótese parcial; Grampos Dentários; Estética Dentária.

\section{AESTHETIC SOLUTIONS FOR REMOVABLE PARTIAL DENTURE RETAINED BY METAL CLASPS}

\begin{abstract}
The construction of a removable partial denture (RPD) aims the replacement of lost dental units, incorporating them into the stomatognathic system, without harming the biomechanical balance. Besides, there is a great challenge today, of combining function and esthetics. The difficulty in achieving esthetic harmony by the presence of metal clasps on labial surfaces of anterior teeth justify studies aimed at solving this problem in the RPDs. There is still the fact that this procedure is simpler and less costly for the patient compared to other treatment options. Therefore, this study presents esthetic solutions for RPD and suggests the comparison among conventional metal clasps on buccal retainers with clips in the palatal area, through three clinical cases of patients with partial absence of anterior dental units. Two prosthetic pieces for each patient were made: 1) prioritizing only the biomechanical principles and 2) considering both the biomechanical and cosmetic principles. It was observed that MDL and Quereilhac clasps are viable alternatives to get esthetics in RPDs of


anterior edentulous patients with Class III and IV of Kennedy. Therefore, it can be concluded that there was an improvement in smile of individuals partially edentulous in the anterior region, by not viewing the metal structure on the buccal area of the teeth.

Keywords: Denture; Partial; Dental Clasps; Esthetics; Dental.

\section{INTRODUÇÃO}

Apesar do conhecimento e da capacidade prática de reabilitar a perda de dentes, a especialidade Prótese dentária encontra como grande desafio, a substituição dos dentes perdidos aliando a função à estética. ${ }^{(1,2)}$ Contudo, a preocupação com a estética não pode interferir na biomecânica da prótese, a ponto de comprometer as estruturas orais remanescentes. ${ }^{(3)}$

A construção de uma prótese para o desdentado parcial, tem por finalidade a reposição das unidades dentárias perdidas, incorporando-as ao aparelho mastigatório, sem ferir o equilíbrio biomecânico imprescindível à preservação das estruturas biológicas que o compõem..$^{(4,5)}$

A Prótese Parcial Removível (PPR) é uma alternativa de reabilitação oral que, além de repor dentes ausentes, reconstitui também tecidos circunvizinhos de uma só vez, sem prejuízo as estruturas remanescentes ${ }^{(6)}$ sendo, menos onerosas em relação às próteses fixas e implantossuportadas, trazendo grande benefício social. ${ }^{(5)}$

Os insucessos de uma PPR podem ser decorrentes de negligência do profissional com relação aos princípios fundamentais que devem reger a construção desse tipo de prótese, de procedimentos laboratoriais incorretos, má colocação por parte do paciente ou da combinação destes fatores. ${ }^{(6)}$

A PPR é um tipo de reabilitação oral que busca restabelecer função, fonética e estética, sendo esta última, a razão de maior procura para a reabilitação. ${ }^{(7)}$ Para se conseguir uma reabilitação mais estética, deve-se incorporar aos trabalhos protéticos detalhes que os tornem imperceptíveis. Assim sendo, quanto menor a possibilidade de notar sua presença, melhores resultados serão obtidos. ${ }^{(3,8)}$

Quando dentes anteriores devem ser utilizados para apoiar as próteses parciais removíveis, as unidades que são visíveis ao sorriso ou fala exibem grande dificuldade de estabelecimento da harmonia estética devido à presença dos grampos metálicos. ${ }^{(9,10)}$

Atualmente existem diversos recursos técnicos capazes de suprir as limitações estéticas da PPR. Os principais descritos na literatura são: PPR e prótese parcial fixa 
conjugada, ${ }^{(1,2,10,11)}$ PPR com eixo rotacional, ${ }^{(3,11)}$ grampos estéticos (grampos de resina $),{ }^{(11,12,13)}$ retenção friccional ${ }^{(14)}$ e grampos metálicos estéticos. ${ }^{(6,11,15,16)}$

A retenção friccional ou retenção por contato parte do princípio que quanto maior o contato entre a prótese e o dente pilar, menor a necessidade de retenção mecânica por meio de grampos. Sendo assim trata-se de um bom artifício em ausências de dentes anteriores, já que aumentando a retenção friccional podem ser excluídos os retentores convencionais que desfavorecem a estética. ${ }^{(14)}$

No entanto, existem grampos que além de conferir a retenção necessária à PPR, favorecem a estética devido a sua área de retenção se restringir à face lingual/palatina. $\mathrm{O}$ grampo circunferencial Quereilhac, como alternativa estética, não é muito relatado na literatura. Trata-se de um retentor extracoronário que necessita da confecção de dois conectores menores nas proximais do dente suporte, dois apoios e um braço por lingual, que faz a retenção da peça. Portanto, o dente se encontra envolvido por mesial e distal, promovendo uma sustentação adequada e conferindo boa relação estética, pois o grampo não deixa estruturas metálicas à mostra por vestibular, o que o faz ser uma boa indicação em determinados casos, principalmente no arco superior. ${ }^{(15)}$

Considerado um dos retentores extracoronários mais estéticos, o retentor MDL modificado está indicado para dentes anteriores e eventualmente para pré-molares se a estética for relevante. Por apresentar apenas um braço, esse retentor deve abraçar mais de $180^{\circ}$ do perímetro da coroa para se obter equilíbrio horizontal das forças. Para isso a ponta ativa deve se localizar próxima ao espaço protético, de forma que a força exercida pelo mesmo seja anulada pela porção inicial do retentor que é rígida. ${ }^{(11)}$

Entendendo-se a disponibilidade de alternativas estéticas na PPR, o objetivo deste trabalho foi apresentar 3 casos clínicos de reabilitação com Próteses Parciais Removíveis retidas por grampo metálicos. Para cada situação foram confeccionadas duas próteses sendo uma atendendo apenas aos princípios biomecânicos e outra considerando tanto aos biomecânicos quanto estéticos.

\section{DESCRIÇÃO DOS CASOS}

Foram realizados três casos clínicos de reabilitação oral com Próteses Parciais Removíveis, no módulo de odontologia da Universidade Estadual do Sudoeste da Bahia (UESB), os pacientes selecionados não possuíam distinção de raça, faixa etária ou gênero e 
apresentavam ausência de unidades dentárias anteriores, podendo ser classificados como Classe III ou IV de Kennedy maxilar, com áreas de modificações.

Foram planejadas e confeccionadas duas peças protéticas para cada paciente, sendo uma atendendo apenas aos princípios biomecânicos, utilizando grampos convencionais e outra considerando tanto os princípios biomecânicos quanto os estéticos, utilizando grampos com braços de retenção apenas por lingual/palatina nos dentes anteriores, ou substituição destes braços retentores por placas proximais, lançando mão assim do aumento da retenção friccional.

\subsection{CASO 01}

Paciente do gênero feminino, 33 anos, apresentava perda parcial dos dentes superiores sendo classificada como Classe III modificação 2 de Kennedy, possuindo a presença das unidades 1.7, 1.3, 2.3, 2.6 e 2.7. Na PPR não estética planejada para este caso foram utilizados quatro grampos simples nos pilares diretos. Para a PPR estética optou-se por dois grampos MDLs modificados nas unidades anteriores e dois grampos simples nos pilares diretos posteriores. (Figuras 1 e 2)

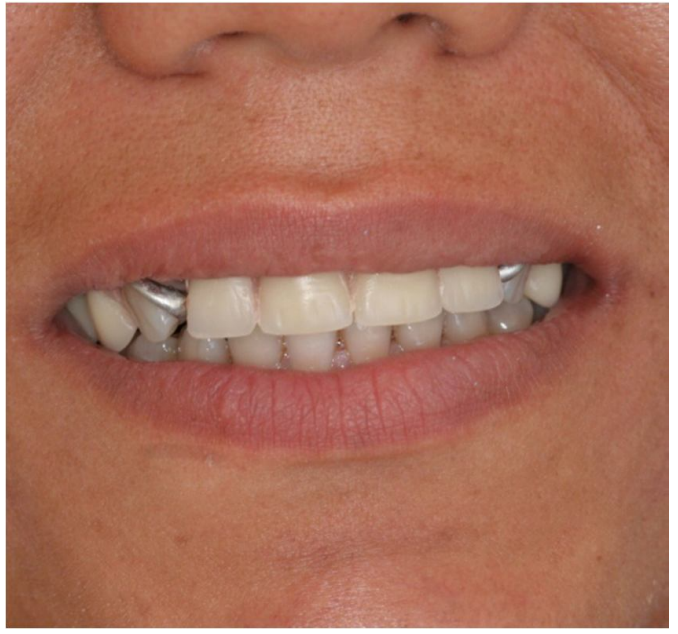

Figura 1 - Vista Frontal do Caso 1 com a PPR não estética instalada

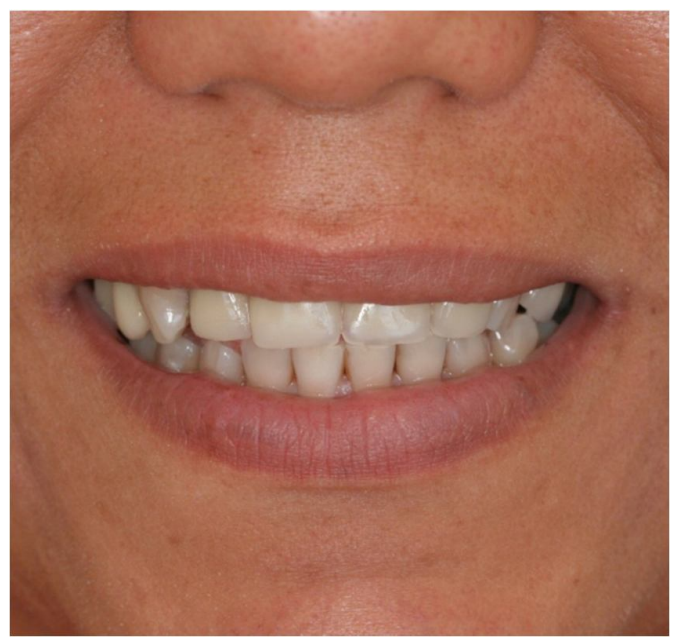

Figura 2 - Vista Frontal do Caso 1 com a PPR estética instalada 


\subsection{CASO 02}

Paciente do gênero feminino, 42 anos, apresentava perda parcial das unidades dentárias superiores sendo classificada como Classe IV de Kennedy, necessitando apenas de reabilitação das unidades $1.2,1.1,2.1$ e 2.2. Para a PPR não estética foram planejados apenas grampos simples para todos os pilares e para a PPR estética foram utilizados os grampos MDL modificado nas unidades pilares 1.3 e 2.3 e grampos simples nas unidades 1.6 e 2.6 . (Figuras 3 a 5)

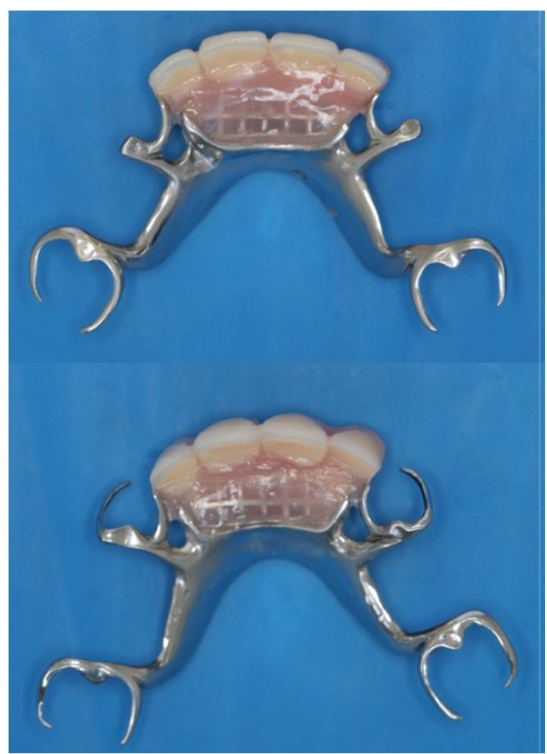

Figura 3 - Vista Oclusal da PPR estética (acima) e da PPR não-estética (abaixo)

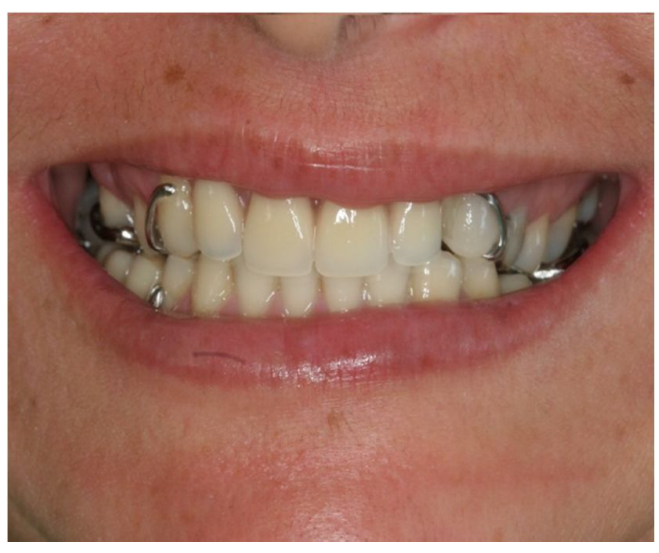

Figura 4 - Vista Frontal do Caso 2 com a PPR não estética instalada

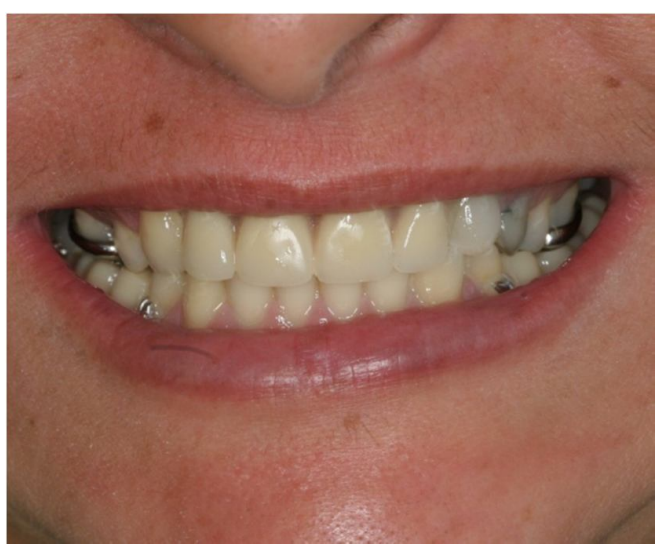

Figura 5 - Vista Frontal do Caso 2 com a PPR estética instalada 


\subsection{CASO 03}

Paciente do gênero feminino, 36 anos, apresentava perda parcial das unidades dentarias superiores sendo classificada como Classe III modificação 2 de Kennedy, tendo presente as unidades dentárias 1.7, 1.6, 1.3, 1.2, 2.2, 2.3 e 2.7. Na PPR não estética foram utilizados grampos simples nas unidades $1.6,1.3,2.3$ e 2.7 e grampos de ação posterior nas unidades 1.2 e 2.2. Para a PPR estética foram utilizados grampos simples nas unidades 1.6 e 2.7, Quereilhac nas unidades 1.3 e 2.3, e apenas retenção friccional (apoio e placa proximal) nas unidades 1.2 e 2.2. (Figuras 6, 7 e 8 )

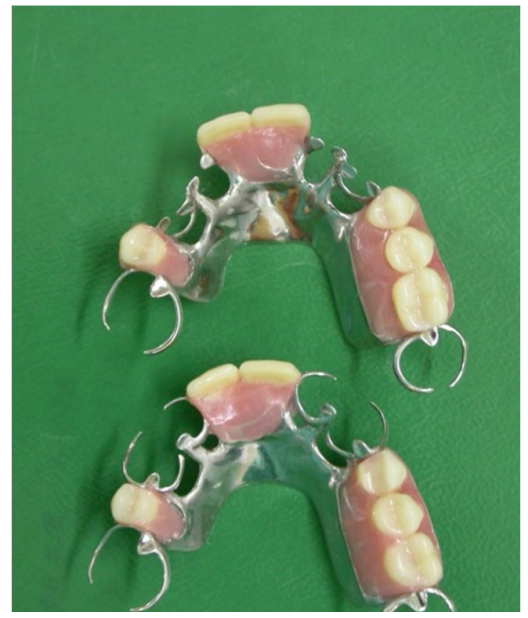

Figura 6 - Vista Oclusal da PPR estética (acima), e da PPR não estética (abaixo)

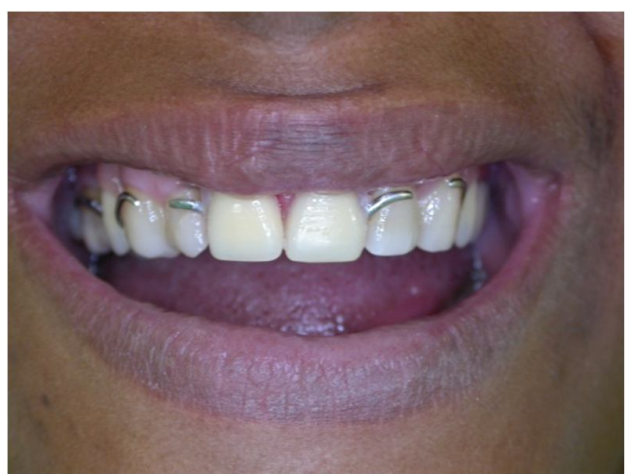

Figura 7- Vista Frontal do Caso 3 com a PPR não estética instalada

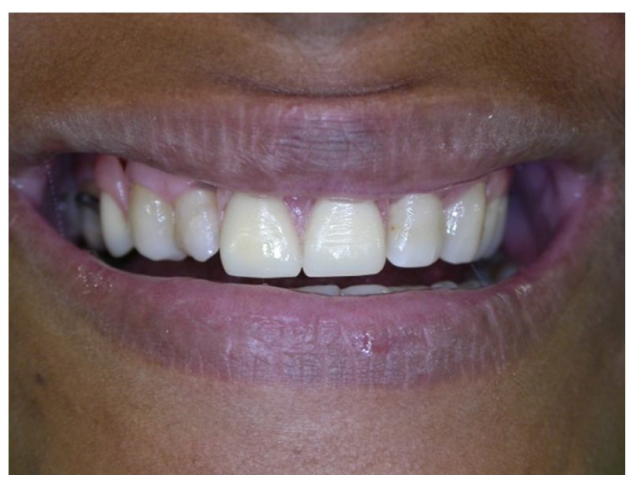

Figura 8 - Vista Frontal do Caso 3 com a PPR estética instalada 


\section{CONCLUSÃO}

O grampo quereilhac, mesmo sendo citado na literatura apenas por Rebossio em 1963, atende aos requisitos necessários para um grampo circunferencial, conferindo à prótese suporte, estabilidade e retenção, com melhora estética significativa. A utilização deste grampo promove maior naturalidade ao sorriso, com o mínimo de metal aparente, pois não existe metal na face vestibular do dente pilar que o recebe. A estabilidade será conferida pela retenção friccional dos conetores menores localizados nas faces proximais.

O grampo MDL modificado também se torna outra alternativa estética. A retenção do mesmo é garantida pela ponta ativa do braço de retenção que está localizado na face proximal do dente pilar, mascarada pela resina acrílica da base da prótese.

Assim, verifica-se que os grampos MDL modificado e Quereilhac são alternativas viáveis para atender o requisito estético em PPRs de pacientes desdentados, cujos arcos são classificados como Classe III e IV de Kennedy.

Todos os pacientes utilizavam anteriormente, próteses parciais mucossuportadas insatisfatórias no aspecto estético. Após a conclusão dos casos as duas novas próteses removíveis foram entregues aos pacientes, ficando a cargo deles a escolha de qual peça utilizar, de acordo com o conceito estético de cada um. Todos fizeram a opção pelas próteses com grampos estéticos. Assim é importante que os planejamentos das PPRs a grampo associe os princípios biomecânicos e estéticos, valorizando a aceitação dos pacientes às reabilitações com próteses parciais removíveis.

\section{REFERÊNCIAS}

1. Santos LB, Carvalho MRT, Vieira TEC, Oliveira AS. Sistemas de encaixe em prótese parcial removível: tendências atuais no município de salvador - Bahia. Int. J. dent. $2005 ;(4): 8-14$.

2. Silva FAP, Ferreira JAND, Ferreira DF. eBook Jubileu de ouro $25^{\circ} \mathrm{CIOSP}$. Reabilitação protética associando prótese fixa e parcial removível; 2007. p. 45-84.

3. Zuim PRJ, Bezzon OL, Sousa V, Garcia AR. Recursos estéticos com próteses parciais removíveis retidas por grampos - eixo rotacional. Revista Odontológica de Araçatuba. 2003; 24:27-32.

4. Rezende AB. Atlas de prótese. Sistema de encaixe. São Paulo: Artes Médicas; 2003. p. 170-189. 
5. Kliemann C, Oliveira W. Manual de prótese parcial removível. São Paulo: Santos; 1999.

6. Todescan R. Atlas de Prótese Parcial Removível. $1^{a}$ ed. São Paulo: Santos; 1996. p. 345.

7. Beaumont, AJ. An Overview of esthetics with removable parcial dentures. Quintessence Internacional. 2002;33:47-755.

8. Dawson PE. Avaliação diagnóstico e tratamento dos problemas oclusais. São Paulo: Artes Médicas; 1980.

9. Cosme DC, Rivaldo EG, Nakamura EM, Frasca LCF. Prótese parcial removível associada à prótese fixa adesiva através de encaixe extracoronário. Rev. odonto ciênc. 2005;20:187-190.

10. Quagliatto PS, Costa MM, Oliveira JE. Sistema total D - Nova Solução Estética para Próteses Parciais Removíveis. RGO. 2000;48:82-4.

11. Costa MM, Strine EM, Costa TCX, Oliveira JEC. Considerações estéticas na construção de uma prótese parcial removível. Revista ibero-americana de prótese clínica \& Laboratorial. 2004;6:199-213.

12. Chee WWL, Cho GC. Achieving esthetics with removable partial dentures. J Calif Dent Assoc. 1990;18:19-22.

13. Puigpelat A, Samson J, Anglada JM, Oortiz E. Nuevo material estético en prótesis partial removible. Polioximetileno o resina acetálica. Odonto Estomatologia. 1993;2:59-63.

14. Brudvik JS, Palacios R. Lingual retention and the elimination of the Visible Clasp Arm. J. Esthet Restor Dent. 2007;19:247-255.

15. Rebossio D. Protesis Parcial Removible como parte del tratamiento del parcialmente desdentados. $3^{\mathrm{a}}$ ed. Buenos Aires: Mundi; 1963. p. 662.

16. Eisenburge, M, Addy, M. Radiological examination of dental castings-a review of the method and comparisons of the equipment. J Oral Rehabil. 2002 jul;29(7):609-14. 